\title{
An efficient recycling process of glycolysis of PET in the presence of a sustainable nanocatalyst
}

Zengwei Guo ${ }^{a}$, Karin Lindqvista ${ }^{a}$, Hanna de la Motte ${ }^{b}$

${ }^{a}$ Swerea IVF, Department of Textile and Plastics, Box 104, SE-431 22, Mölndal, Sweden

${ }^{\mathrm{b}}$ RISE Research Institutes of Sweden, Division Bioeconomy, Box 24036, SE-400 22 Göteborg, Sweden

Correspondence to: Zengwei Guo (E-mail: Zengwei.guo@swerea.se)

\begin{abstract}
In this paper it demonstrates that the catalyst Perkalite F100 efficiently works as a nanocatalyst in the depolymerization of poly(ethylene terephthalate)(PET). After depolymerization of PET in the presence of ethylene glycol and the Perkalite nanocatalyst, the main product obtained was bis(2-hydroxylethyl) terephthalate (BHET) with high purity, as confirmed by FTIR and NMR. The BHET monomers could directly serve as starting materials in a further polymerization into PET with a virgin quality, and contribute to a circular solution of the vast disposal of PET polymers to the environment. Compared with the direct glycolysis of PET, the addition of a pre-degradation step was shown to reduce the reaction time of reaching the depolymerization equilibrium. Additionally, the addition of the pre-degradation step allowed lower reaction temperatures. Therefore, the strategy to include a pre-degradation step before depolymerization is suitable to increase the efficiency of the glycolysis reaction of PET into BHET monomers.
\end{abstract}




\section{INTRODUCTION}

Over the last a few decades, the consumption of poly(ethylene terephthalate) (PET) has tremendously increased. Today, PET is the fourth most produced polymer in the world, after polyethylene (PE), polypropylene (PP) and polystyrene (PS). The success of PET arises from its excellent mechanical properties, processability and low price. These properties make PET suitable for several applications, especially for fiber applications. The PET fiber is the most common fiber in textile industry nowadays. Every year, it is estimated that approximately 70 million barrels of oil are used in the manufacturing of virgin polyester fibers worldwide. ${ }^{1}$ However, there are many issues regarding these fibers, such as consumption of petroleum resources to produce the fibers, the astonishing volume of disposed waste and the $\mathrm{CO}_{2}$ emissions related to both the production and disposal of the fibers. To save energy and protect our living environment, recycling of post-consumer PET is necessary.

To recycle the polyester PET, the concept of chemical depolymerization of PET into its original feedstock monomers, and then reuse these monomers for virgin polymer synthesis, has been intensively studied over the last few decades. Several depolymerization methods have been investigated to break the ester bonds in PET chains, such as methanolysis, ${ }^{2}$ glycolysis, ${ }^{3}$ hydrolysis, ${ }^{4}$ aminolysis etc. ${ }^{5}$ Among these methods, glycolysis is reported to be an important recycling method due to its low cost and energy consumption. ${ }^{6,7}$ However, glycolysis of PET in the absence of catalyst is an extremely slow process. Consequently, there has been a strong interest in the development of highly efficient catalysts for the glycolysis of PET. It has been found that $\mathrm{Zn}^{2+}$ containing salts and mixed oxides show very high catalytic efficiency. ${ }^{8,9,10}$ However, $\mathrm{Zn}^{2+}$ is a heavy metal ion, and catalysts containing $\mathrm{Zn}^{2+}$ might have a potential of 
generating secondary pollution to the environment. To avoid this problem, sodium carbonate and sodium bicarbonate were studied as alterative catalysts to zinc catalysts in the depolymerization of PET waste. Different from $\mathrm{Zn}^{2+}$ containing catalyst, sodium carbonate and sodium bicarbonate are eco-friendly since sodium cation has less severe environmental impact than that of $\mathrm{Zn}^{2+}$. It was found that the depolymerization efficiency of these two catalysts was close to that of zinc acetate. ${ }^{11,12}$ Recently, the catalytic capacities of ionic liquids (ILS) have been investigated, and in particular as green transesterfication catalysts in the glycolysis of PET. ${ }^{13,14}$ However, the high catalyst concentration and long reaction time are challenges for the application of ILs as catalysts in the large-scale glycolysis of PET. To realize a large-scale recycling of PET by glycolysis, a cheap, efficient and environmentally friendly catalyst would be necessary.

Recently, we found that Perkalite F100 works very efficiently as a transesterification catalyst in the alcoholysis of polyester. Perkalite F100 is a synthetic nanoclay, containing Mg-Al layered double hydroxides. Different from the catalysts containing a $\mathrm{Zn}^{2+}$ cations, the synthetic nanoclay is environmentally benign, and might even be directly disposed into the natural environment. ${ }^{15,} 16,17$ Moreover, the synthetic nanoclay has a layered structure in the nanometer scale level. As the catalyst, such structure enables nanoclay to possess a very high active surface, which can significantly enhance its catalytic effect. ${ }^{17}$

However, nanocatalysts are prone to form aggregations in reaction systems, which leads to decreasing their catalytic performance. To improve depolymerization efficiency, a "predegradation step" was developed in this study. In the designed "pre-degradation step", 
Perkalite nanocatalyst was first mixed with PET by a melt compounding process and then a small amount of ethylene glycol (EG) was added to the compounder. During the compounding, the nanocatalyst together with EG triggered the degradation of PET. After compounding, the obtained PET containing the nanocatalyst was added to a reactor for further glycolysis into monomers. In this study, we investigated the feasibility of using Perkalite F100 as nanocatalyst in the depolymerization of PET. In addition, the influences of the pre-degradation step in the whole depolymerization process were investigated.

\section{EXPERIMENTAL}

\section{Materials}

Fiber grade PET was purchased from INVISTA with the brand name of RT20. RT20 is unformulated PET and provided in the form of white pellets. EG with the purity of $99.8 \%$ was bought from Sigma-Aldrich. Perkalite F100 was supplied by AkzoNobel. BHET with a purity of 94.5\% was bought from Sigma-Aldrich. Zinc acetate and sodium bicarbonate were also bought from Sigma-Aldrich.

A stainless steel cylinder was used as the depolymerization reactor in this study as shown in Figure 1. The cylinder was bought from Swagelok. The volume of the cylinder is $150 \mathrm{ml}$ and the material of cylinder is stainless steel 316L.

\section{Pre-degradation step}

PET was first dried at $120^{\circ} \mathrm{C}$ over night and then compounded with Perkalite F100 in Brabender AEV 651 from Svenska Keraminstitutet. The temperature of Brabender was set to be $260^{\circ} \mathrm{C}$ and the rotation speed was $80 \mathrm{rpm}$. After compounding PET with Perkalite nanocatalyst for $1 \mathrm{~min}$, a 
certain amount of EG was slowly injected into the Brabender within $1 \mathrm{~min}$, and then the compounding was continued for another $2 \mathrm{~min}$.

\section{Glycolysis}

The PET sample (pre-degradation PET or virgin PET) were cryogenically grinded into fine powder before the reaction. PET powder $(10 \mathrm{~g})$ and EG $(50 \mathrm{~mL})$, and catalysts in case of the direct glycolysis, were added into the depolymerization reactor. The air inside the reactor was purged by nitrogen before closing the reactor. Then, the reactor was put into an oven at desired temperature for a certain time. The rotation of the reactor inside the oven was $1 \mathrm{rpm}$. When the reaction was finished, the reactor was quenched in cold water. The reaction products in the reactor were mixed with $200 \mathrm{ml}$ of hot deionised water. The obtained slurry from reactor was heated up to the boiling point and then filtered immediately. After the first filtration, some white floccules appeared in the filtrate, and the filtrate was thus filtered a second time. The filter cakes obtained from the two filtrations were dried at $100^{\circ} \mathrm{C}$ for $8 \mathrm{~h}$ and then weighed as oligomers. After that, the filtrate from the second filtration was kept in $4^{\circ} \mathrm{C}$ overnight. During the storage time, the BHET crystallized in the filtrate. The white crystals was collected by filtration and weighed as monomer BHET after drying at $80^{\circ} \mathrm{C}$ overnight. The molar yield of BHET was calculated according to Equation 1

Yield of BHET $(\mathrm{mol} \%)=\frac{\frac{w_{B H E T}}{M_{B H E T}}}{\frac{w_{P E T}}{M_{P E T}}} \times 100 \%$

Where $\mathrm{M}_{\mathrm{BHET}}$ is $245 \mathrm{~g} / \mathrm{mol}$ and $\mathrm{M}_{\mathrm{PET}}$ for the repeating unit is $192 \mathrm{~g} / \mathrm{mol}$.

\section{Molecular weight measurement}


The molecular weight (Mw) of PET was measured by a GPC method. PET was first dissolved in a 1,1,1,3,3,3-hexafluoro-2-propanol (HFIP)/sodium trifluoroacetate ( $5 \mathrm{mM}$ ) mixed solution. The sample concentration was $1 \mathrm{mg} / \mathrm{ml}$, and the measured temperature was $40^{\circ} \mathrm{C}$. To calculate the molecular weight distributions, poly(methyl methacrylate) (PMMA) was used as standard.

\section{Differential scanning calorimetry (DSC)}

DSC was carried out with DSC 1 from Mettler Toledo under nitrogen atmosphere. The temperature scans were carried out between $30^{\circ} \mathrm{C}$ and $280^{\circ} \mathrm{C}$ at a heating rate of $10^{\circ} \mathrm{C} / \mathrm{min}$.

\section{Fourier Transform Infrared Spectroscopy (FTIR)}

FTIR analysis was performed on a Perkin Elmer Spectrum One spectrometer, from $400 \mathrm{~cm}^{-1}$ to $4000 \mathrm{~cm}^{-1}$, with a resolution of $4 \mathrm{~cm}^{-1}$.

\section{Nuclear Magnetic Resonance Spectroscopy (NMR)}

${ }^{1} \mathrm{H}$ NMR spectra were obtained from a Bruker Advance 400 spectrometer operating at $400 \mathrm{MHz}$. The solvent used to dissolve BHET was DMSO- $\mathrm{d}_{6}$.

\section{RESULTS AND DISCUSSION}

Feasibility of using Perkalite as catalyst in the depolymerization of PET

In our study, we found that Perkalite based on only Mg-Al hydroxides possesses a very high catalytic effect in the depolymerization of PET. As presented in Figure 2, Perkalite nanoclay works very efficiently as catalyst in the glycolysis of PET. At $240^{\circ} \mathrm{C}$, no BHET was obtained in the absence of catalyst. However, when 0.1 wt\% of Perkalite was added in the reaction system, the yield of BHET increased from 0 to 77 mol\%. As the concentration of Perkalite nanocatalyst 
increased, the yield of BHET continued to increase, until a reached maximum value at a Perkalite concentration of $0.5 \mathrm{wt} \%$. When the Perkalite concentration was further increased, the yield of BHET showed a decrease. The decrease of BHET is probably due to the reformation of oligomers from BHET. ${ }^{3}$ As shown in Figure $2 b$, the yield of oligomers first decrease and then increase as the increase of catalyst concentration from $0.1 \mathrm{wt} \%$ to $3 \mathrm{wt} \%$. The minimum yield of oligomer was obtained at the Perkalite concentration of $0.5 \mathrm{wt} \%$. This is consistent with the yield of BHET.

A comparison of the catalytic efficiency of Perkalite nanocatalyst and sodium bicarbonate in the glycolysis of PET was also studied. In these experiments, the conventional catalyst zinc acetate was used as a reference. As shown in figure 3, the Perkalite nanocatalyst has the highest catalytic efficiency. The yield of BHET was over 80 mol\%. Compared with Perkalite nanocatalyst, sodium bicarbonate shows a slightly lower catalytic efficiency. However, its catalytic efficiency is almost the same as that of zinc acetate, which is consistent with the literature results. ${ }^{11,12}$

\section{Influences of pre-degradation step in the glycolysis of PET}

Kurokawa et al. suggest that the methanolysis of PET in the absence of the catalyst includes a three-step mechanism, according to their GPC analysis. ${ }^{2}$ In the first step, PET chains are shorted to around one-third. This step takes place easily and fast. The PET chains might be cut off from tie molecules since these molecules are in the amorphous part. In the second stage, PET is further shorted to oligomers. However, this stage is a slow process. Glycolysis of PET into oligomers in shorter than 30 min of reaction time seems to be difficult according to litterature. ${ }^{18,19}$ Under certain circumstances, the generation of oliogmer can be accelerated, for 
example addition of a co-solvent which can dissolve or swell the PET crystal parts. ${ }^{2,20}$ The last stage is the conversion of PET oligomers to monomer BHET. The presence of catalyst is very important to achieve high yield of BHET in this step. ${ }^{2}$

In our beginning experiments with Perkalite, the nanocatalyst, EG and PET were directly added into the reactor. After the reaction, it was found that Perkalite nanocatalyst tended to form aggregations. The formation of aggregations probably reduces the catalytic surface of Perkalite and thus the catalytic efficiency. To eliminate the possibility of aggregations forming in the reaction system, a new process was developed. In this process, Perkalite nanocatalyst was first compounded with PET in a Brabender mixer to obtain a homogenous dispersion of nanocatalyst in the PET matrix. The homogenous dispersion of nanocatalyst in PET would facilitate the following glycolysis reaction since EG molecules will have a higher chance to access both active sites of the nanocatalyst and the ester bond of PET in the reaction system. In addition, the system was designed so that a small amount of EG could slowly be injected into the Brabender mixer during the compounding. Based on Kurokawa's theory, the injection of EG probably triggers the first and second stage of degradation of PET within a short compounding time in the Brabender. In this study, the designed compounding step with the injection of EG is called "pre-degradation step", and it was found that this pre-degradation step significantly influences the glycolysis process.

\section{Molecular weight variation}

As shown in the DSC thermograms (Figure 4A), the virgin PET showed a melting peak at $258^{\circ} \mathrm{C}$. When PET was compounded with only Perkalite nanocatalyst, the melt temperature of PET was 
not changed after the compounding. However, when EG was injected into the Brabender, PET started to degrade. As revealed in Figure 4B, after compounding PET with Perkalite nanocatalyst and $2 \mathrm{ml} \mathrm{EG}$, the melting peak of PET decreased to $252^{\circ} \mathrm{C}$. In addition, the peak became broad. This indicates that virgin PET degrades into various PETs with lower molecular weights. When the injected amount of EG increased to $6.5 \mathrm{ml}$, the melting peak of PET was further decreased to $238^{\circ} \mathrm{C}$, which is $20^{\circ} \mathrm{C}$ lower than that of virgin PET. It implies that PET degrades into low molecular weight oligomers. ${ }^{21}$

To further confirm the degradation of PET, GPC analysis of PET before and after compounding with nanocatalyst and EG was conducted. The results are listed in Table 1. Compared with starting PET, the molecular weight of pre-degraded PET showed a dramatic decrease after compounding with EG and Perkalite nanocatalyst. The weight average molecular weight (Mw) of pre-degraded PET was only around 3800, indicating PET has degraded into oligomers with around 20 repeat units.

\section{Glycolysis time and temperature of PET after the pre-degradation step}

The treatment of PET with a pre-degradation step significantly increased the depolymerization speed. As shown in Figure 5, no BHET monomer was obtained after 20 min the depolymerization temperature of $240^{\circ} \mathrm{C}$. However, when the reaction time increased to $30 \mathrm{~min}$, the yield of BHET was 52 mol\% for PET with a pre-degradation step, while the yield of BHET without a pre-degradation process (i.e. adding the nanocatalyst directly to the reactor) was only $18 \mathrm{~mol} \%$. 
As the reaction time increased to $45 \mathrm{~min}$, the yield of BHET with a pre-degradation step was still twice as high as that of PET without a pre-degradation step. As the time further increased, the yield of BHET did not increase very much. This implies that the depolymerization of PET using a pre-degradation step have reached an equilibrium after a reaction time of around $45 \mathrm{~min}$, and PET sample was completely depolymerised into monomers and small oligomers. However, for the PET without a pre-degradation step, $60 \mathrm{~min}$ is needed to reach the same depolymerization equilibrium. Therefore, the addition of the pre-degradation step before the depolymerization reaction can significantly improve depolymerization speed and thus reduce the reaction time.

In addition, the temperature which can trigger the depolymerization, could also be decreased when PET is treated with a pre-degradation step. As shown in Figure 6a, PET with a predegradation step shows a higher yield of BHET than that of PET without a pre-degradation step in the temperature range of $200-225^{\circ} \mathrm{C}$. Especially, at the reaction temperature at $200^{\circ} \mathrm{C}$, the depolymerization of PET without a pre-degradation step could not be triggered. While, for PET with a pre-degradation step, the depolymerization started at $200^{\circ} \mathrm{C}$. After $1 \mathrm{~h}$ reaction time, the yield of BHET was as high as $25 \mathrm{~mol} \%$. This indicates that the addition of the pre-degradation step allows lower depolymerization temperatures.

\section{Analysis of main product}

FTIR

To confirm the formation of BHET after glycolysis, FTIR analysis was performed. As a reference spectrum, the FTIR spectrum of commercial BHET from Sigma-Aldrich was recorded. As shown in Figure 7, BHET isolated from the depolymerization processes showed similar spectra as the 
BHET reference spectrum. The absorption peaks at $3340 \mathrm{~cm}^{-1}$ and $1135 \mathrm{~cm}^{-1}$ indicate the presence of hydroxyl groups (-OH). The absorption peaks at $1716 \mathrm{~cm}^{-1}, 1253 \mathrm{~cm}^{-1}$ and $1072 \mathrm{~cm}^{-1}$ are signed to the $\mathrm{C}=\mathrm{O}$ band, $\mathrm{C}-\mathrm{O}$ ester bond asymmetric vibration and $\mathrm{C}-\mathrm{O}$ ester bond symmetric vibration, respectively. The absorption peaks between $900 \mathrm{~cm}^{-1}$ to $675 \mathrm{~cm}^{-1}$ are attributed by the vibration of aromatic ring. In addition, the FTIR peaks in all three spectra are quite sharp. This indicates that the purity of obtained BHET from depolymerization process is very high.

DSC

Furthermore, the DSC curve of BHET obtained from depolymerization process with a predepolymerization step shows a sharp endothermic peak at temperature of $111^{\circ} \mathrm{C}$ (Figure 8), which is consistent with the known melting temperature of BHET. ${ }^{22}$

${ }^{1} H N M R$

${ }^{1} \mathrm{H}$ NMR spectrum of BHET obtained from glycolysis process with a pre-degradation step is shown in Figure 9. As a reference, commercial BHET was also recorded. As shown in Figure 7, the two BHET spectra show very similar NMR signals. The signal at $\delta 3.7 p p m$ (peak B) represents the protons of the methylene of $-\mathrm{CH}_{2}-\mathrm{OH}$ group, the signals at $\delta 4.3 \mathrm{ppm}$ (peak $\mathrm{C}$ ) and 4.9ppm (peak $A$ ) indicate the protons of the methylene close to $\mathrm{COO}$ - group and the proton in hydroxyl group. The signal at $\delta 8.1 \mathrm{ppm}$ (peak D) is characteristic of the four aromatic protons. The NMR spectra consistent with those reported in the literature. ${ }^{20}$

\section{CONCLUSIONS}


The feasibility of using Perkalite F100 as a nanocatalyst in the glycolysis of PET has been investigated. It was found that Perkalite F100 showed a very high catalytic activity in the glycolysis of PET. At a nanocatalyst concentration as low as $0.1 \mathrm{wt} \%$, PET can be totally depolymerized into BHET and small oligomers. Compared with sodium bicarbonate and zinc acetate, the Perkalite nanocatalyst shows the highest catalytic efficiency. In addition, Perkalite nanocatalyst is environmentally benign and cheap, which make it suitable as catalyst in the chemical recycling of PET.

The design of a pre-degradation step in the depolymerization process proved to decrease the reaction time to reach depolymerization equilibrium, and allowed lower reaction temperatures. Therefore, it can be concluded that Perkalite F100, especially when introduced in a predegradation step, results in a more efficient glycolysis of PET into BHET.

\section{ACKNOWLEDGEMENTS}

This work was financially supported by Mistra - the Swedish Foundation for strategic Environmental Research. The authors also thank Dr. Jasna Stevanic Srndovic at RISE for NMR analysis. 


\section{REFERENCES AND NOTES}

1. https://oecotextiles.wordpress.com/2013/02/06/antimony-in-fabrics (accessed October 8, 2017)

2. Kurokawa, H.; Ohshima, M.; Sugiyama, K.; Miura, H. Polym. Degrad. Stab. 2003, 79, 529-533.

3. Imran, M.; Kim, B.; Han, M.; Cho, B. G.; Kim, D. H. Polym. Degrad. Stab. 2010, 95, 1686-1693.

4. Palme, A.; Peterson, A.; De la Motte, H.; Theliander, H.; Brelid H. Textiles and Clothing Sustainability 2017, 3:4.

5. Paszum, D. and Spychaj T. Ind. Eng. Chem. Res. 1997, 36, 1373.

6. Lorenzetti, C.; Manaresi, P.; Berti, C.; Barbiroli, G. J. Polym. Environ. 2006, 14, 89-101.

7. Sinha, V.; Patel M. R.; Patel J. V. J. Polym. Environ. 2010, 18, 8-25.

8. Khoonkari, M.; Haghighi, A. H.; Sefidbakht, Y.; Shekoohi, K.; Ghaderian, A. Int. J. Polym. Sci. 2015, 1-11.

9. Syariffudden, A., Norhafizah A. and Salmiaton A. Int. J. Eng. Res. Tech. 2012, 10, 1-8.

10. Imran, M.; Kim, D.; Al-masry, W.; Mahmood, A.; Hassan, A.; Haider, S.; Ramay, S. Polym. Degrad. Stab. 2013, 98, 904-915.

11. Shukla S.R.; Kulkarni K.S. J. Appl. Polym. Sci., 2002, 85, 1765-1770.

12. López-Fonseca R.; Duque-Ingunza I.; Rivas B. de; Arnaiz S.; Gutiérrez-Ortiz J.I. Polym. Degrad. Stab. 2010, 95, 1022-1028.

13. Wang, Q.; Lu, X.; Zhou, X.; Zhu, M.; He, H.; Zhang, X. J. Appl. Polym. Sci. 2013, 129, 3574-3581.

14. Yue, Q.; Yang, H.; Zhang, M.; Bai, X. Polymers 2013, 5, 1258-1271.

15. Xie W., Peng H., Chen L., J. Mol. Catal. A: Chem. 2006, 246, 24-32.

16. Baskaran, T.; Christopher, J.; Ajithkumar, T.G.; Sakthivel, A. Appl. Catal. A 2014, 488, 119-127.

17. Basahel, S.; Al-Thabaiti, S.; Narasimharao, K.; Ahmed, N.; Mokhtar, M. J. Nanosci. Nanotech. 2014, 1931-1946.

18. Imran, M.; Kim, D.; Al-masry, W.; Mahmood, A.; Hassan, A.; Haider, S.; Ramay, M. Polym. Degrad. Stab. 2013, 98, 904-915.

19. Ghaemy, M.; Mossaddegh K. Polym. Degrad. Stab. 2005, 90, 570-576.

20. Liu, F.; Cui, X.; Yu, S.; Li, Z.; Ge, X. J. Appl. Polym. Sci. 2009, 114, 3561-3565.

21. Kao, C. Y.; Cheng, W.H.; Wan, B.Z. Thermochimica Acta 1997, 292, 95-104.

22. Yue, Q.F.; Wang, C.X.; Zhang, L.N.; Ni, Y.; Jin, Y.X. Polym. Degrad. Stab. 2011, 96, 399-403. 


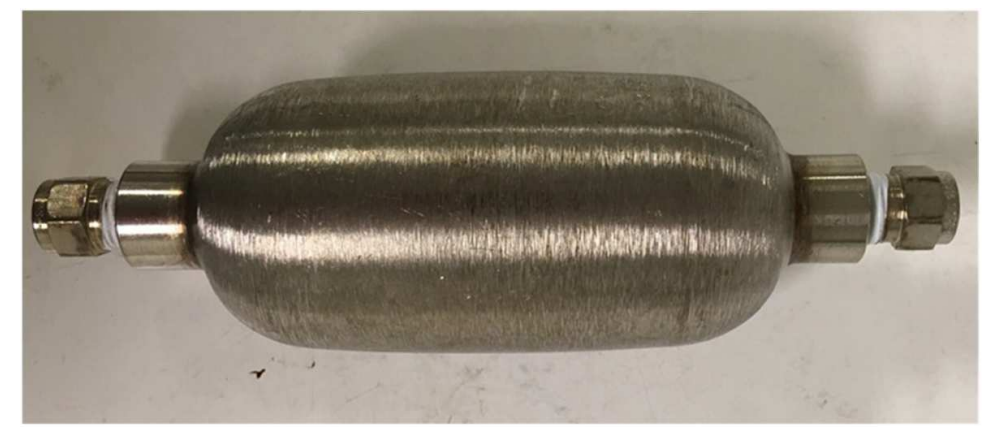

FIGURE 1 Depolymerization reactor used in this study. 


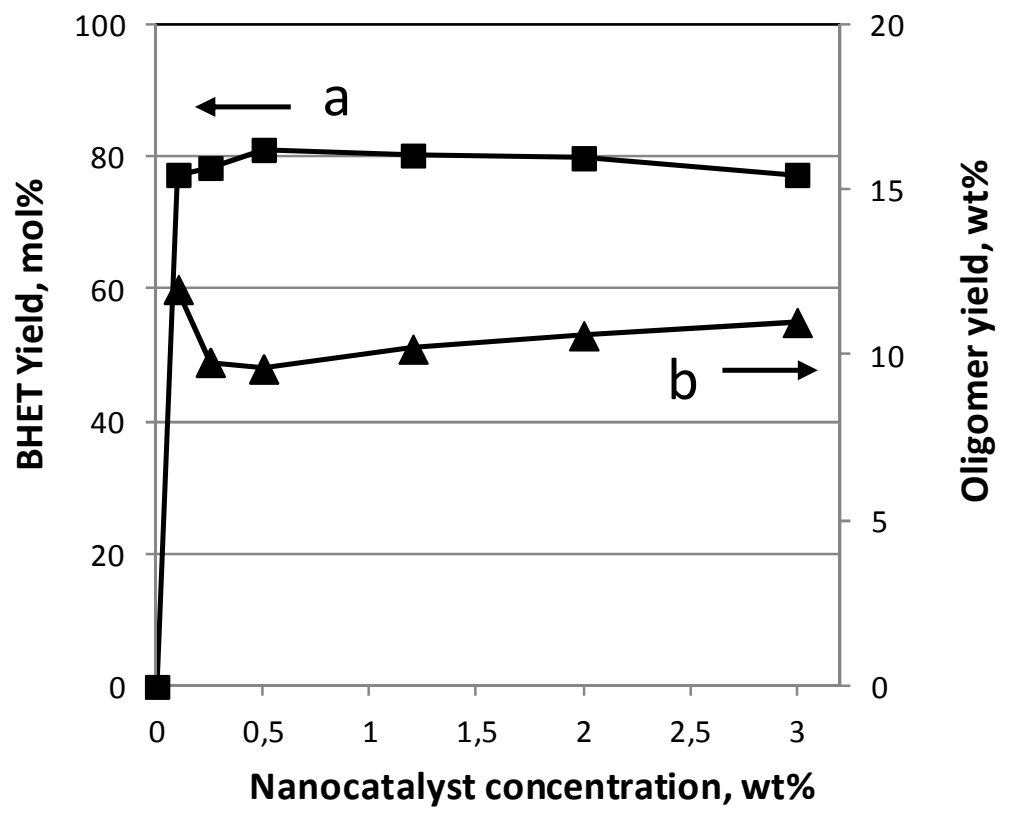

FIGURE 2 Yield of BHET and oligomers as a function of nanocatalyst concentration: $a$ is the yield of BHET, and $b$ is the yield of oligomer. PET, nanocatalyst and EG were directly put into the autoclave. The depolymerization started at $240^{\circ} \mathrm{C}$ and reaction time was $2 \mathrm{~h}$. 


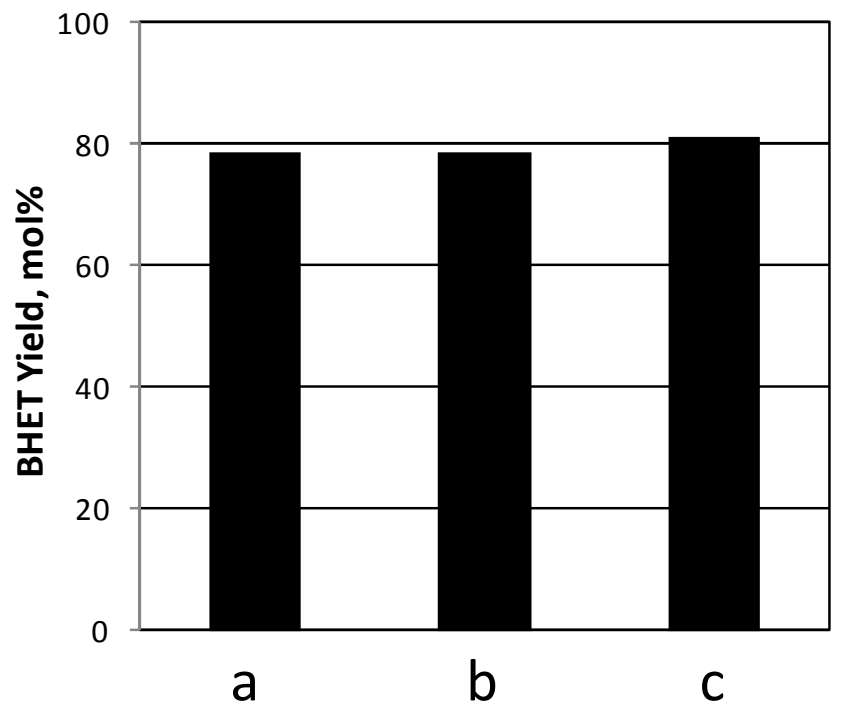

FIGURE 3 Comparison of catalytic efficiency of different catalysts. The catalytic efficiency is expressed in terms of BHET yield. Catalyst in a is zinc acetate. Catalyst in b is sodium bicarbonate. Catalyst in $\mathrm{c}$ is Perkalite nanocatalyst in this study. All the depolymerization reactions were carried out under the same conditions: catalyst concentration was $0.5 \mathrm{wt} \%$; reaction temperature was $240^{\circ} \mathrm{C}$; reaction time was $2 \mathrm{~h}$ and ratio of EG to PET was 5. 

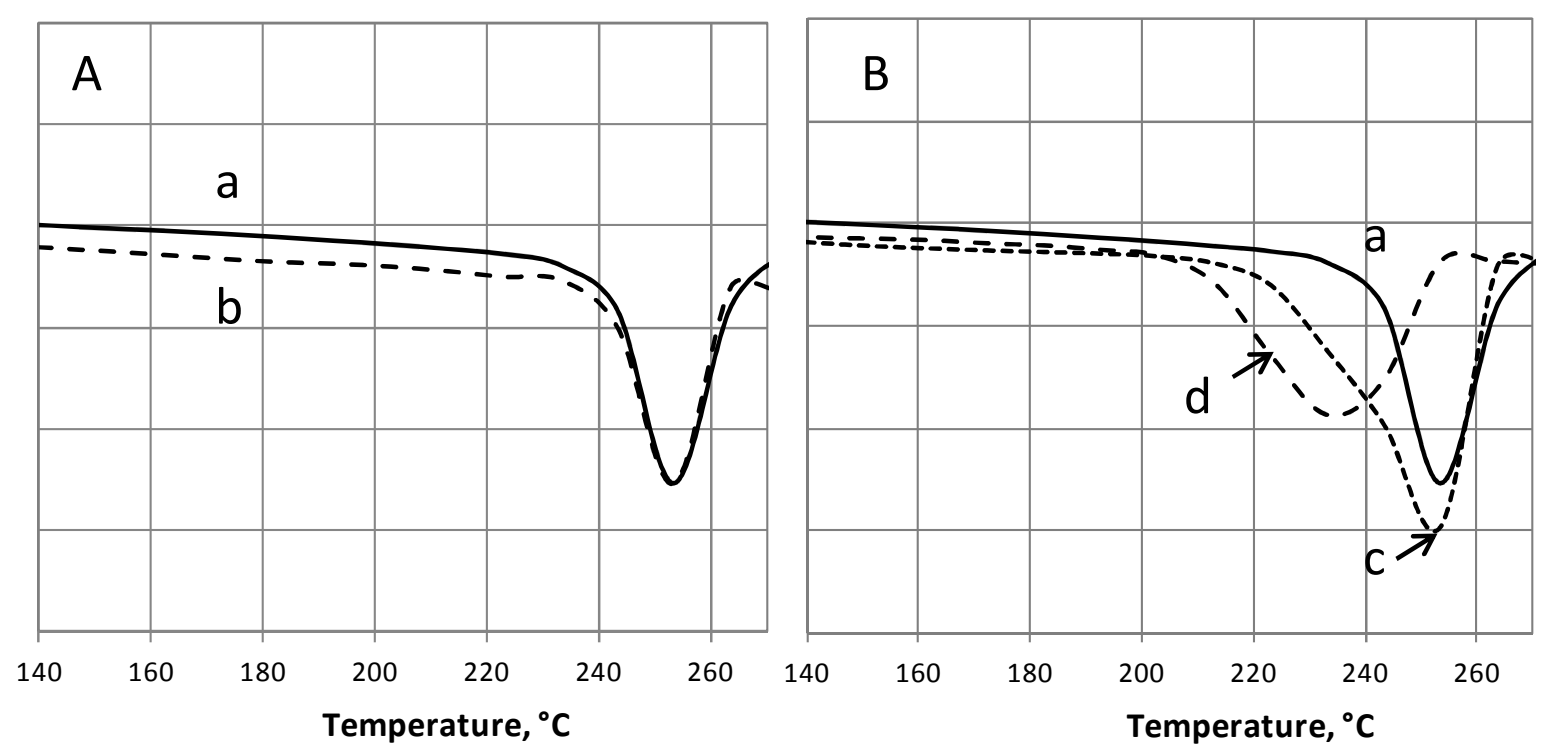

FIGURE 4 DSC curves of PETs: $a$ is virgin PET, $b$ is PET compounded with 0.5 wt\% of nanocatalyst, $c$ is PET compounded with $0.5 \mathrm{wt} \%$ of nanocatalyst and $2 \mathrm{ml}$ of EG injected into the Brabender during the compounding, and d is PET compounded with $0.5 \mathrm{wt} \%$ of nanocatalyst and $6.5 \mathrm{ml}$ of EG injected into the Brabender during the compounding. 


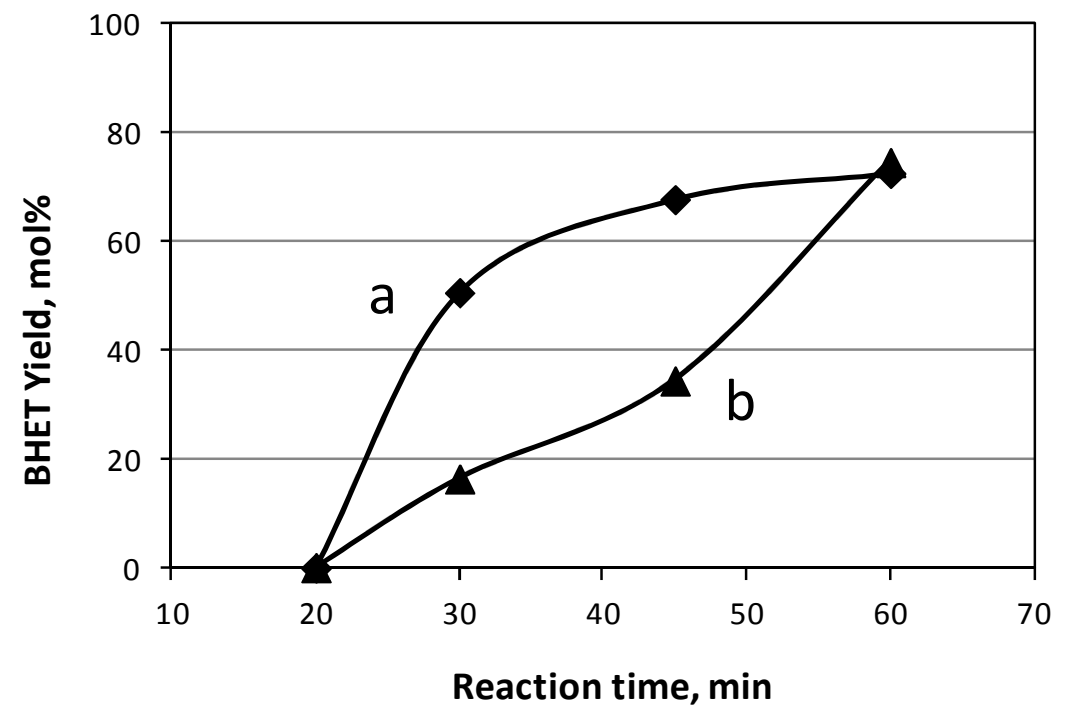

FIGURE 5 BHET yield as function of reaction time: a shows samples with a pre-degradation process, and b shows samples without a pre-degradation process. The reaction temperature for all samples was $240^{\circ} \mathrm{C}$. 


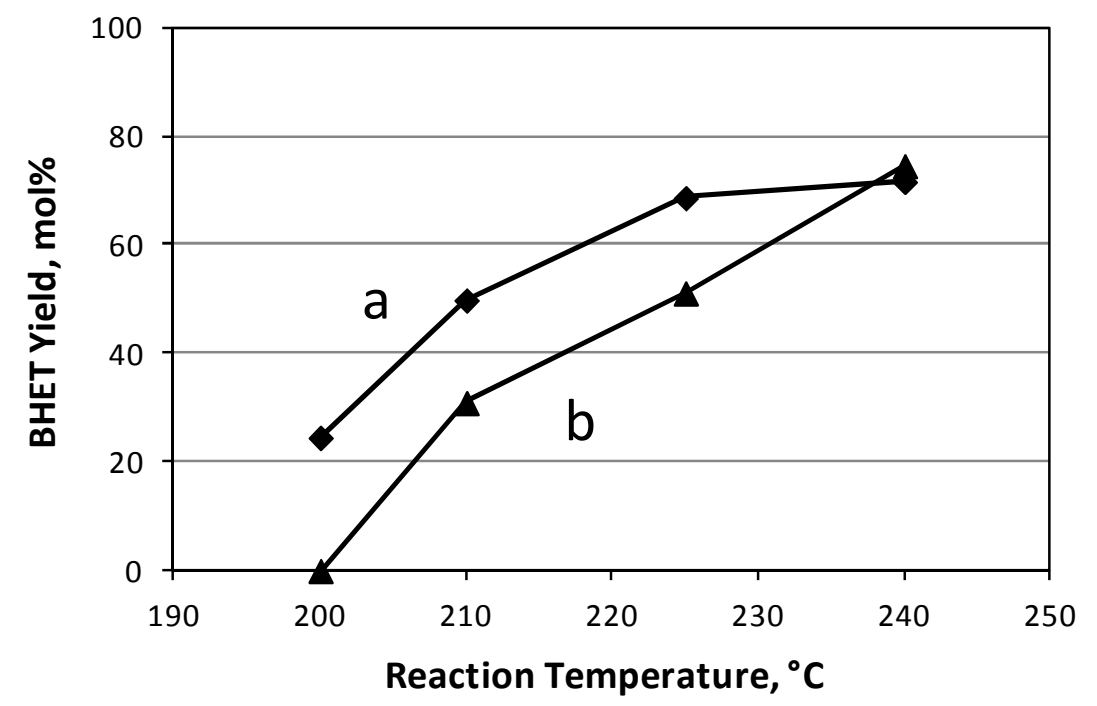

FIGURE 6 BHET yield as function of reaction temperature: a shows samples with a pre-degradation process, and $b$ shows samples without a pre-degradation process. The reaction time for all samples was $1 \mathrm{~h}$. 


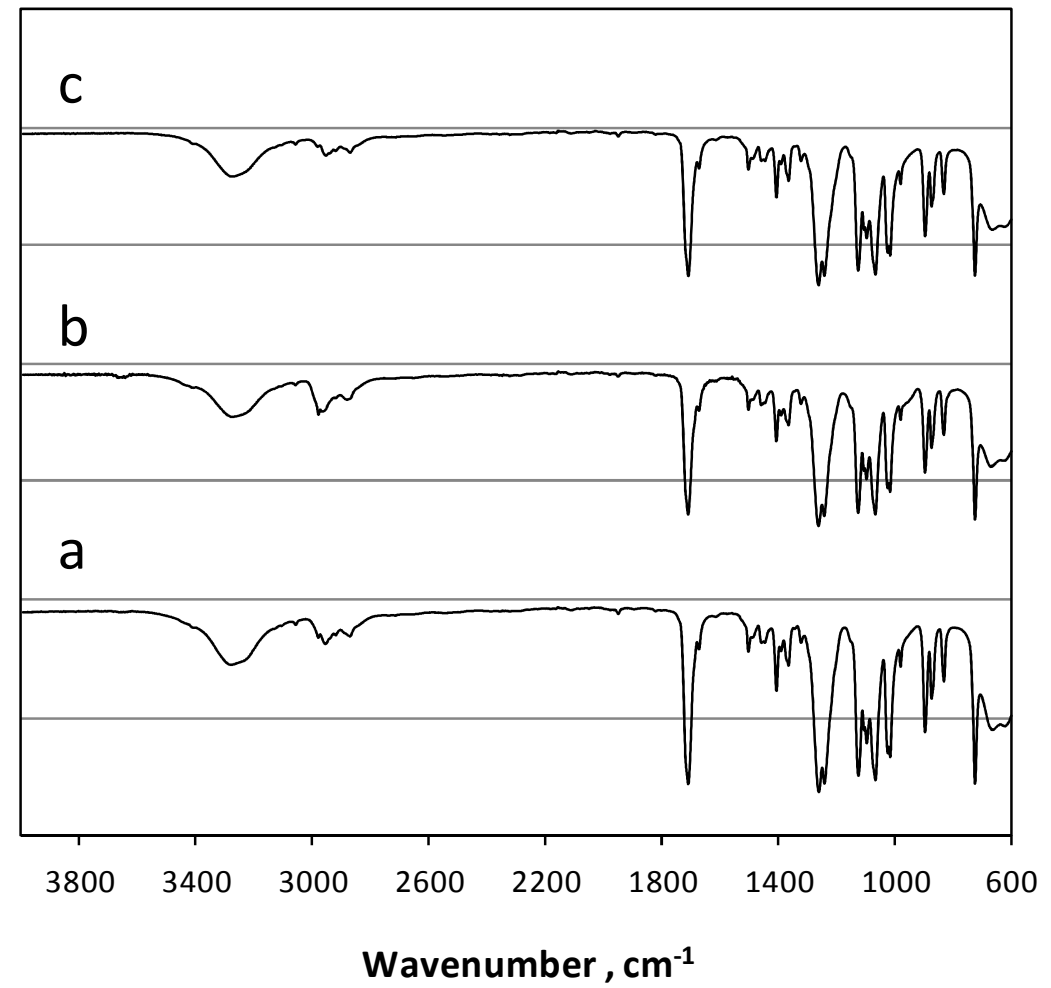

FIGURE 7 FTIR curves of BHETs: $a$ is BHET from Sigma-Aldrich, $b$ is BHET obtained from a depolymerization process without a pre-degradation step, and c is BHET obtained from a depolymerization process with a pre-degradation step. 


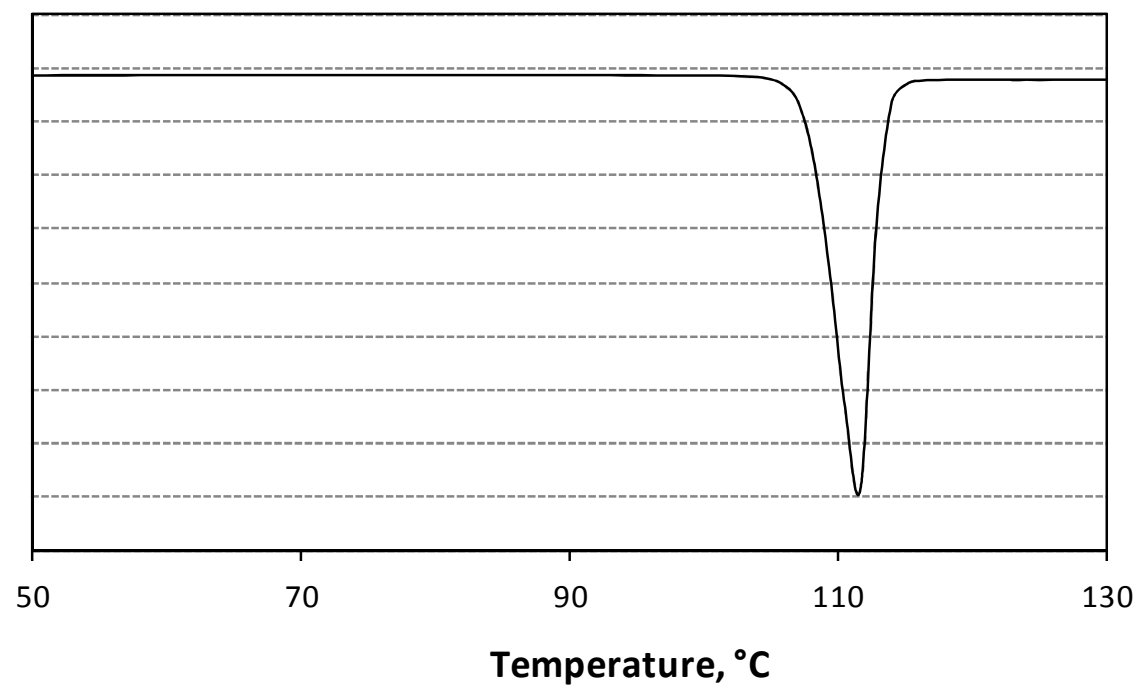

FIGURE 8 DSC curve of BHET obtained from depolymerization process with a pre-degradation step. 


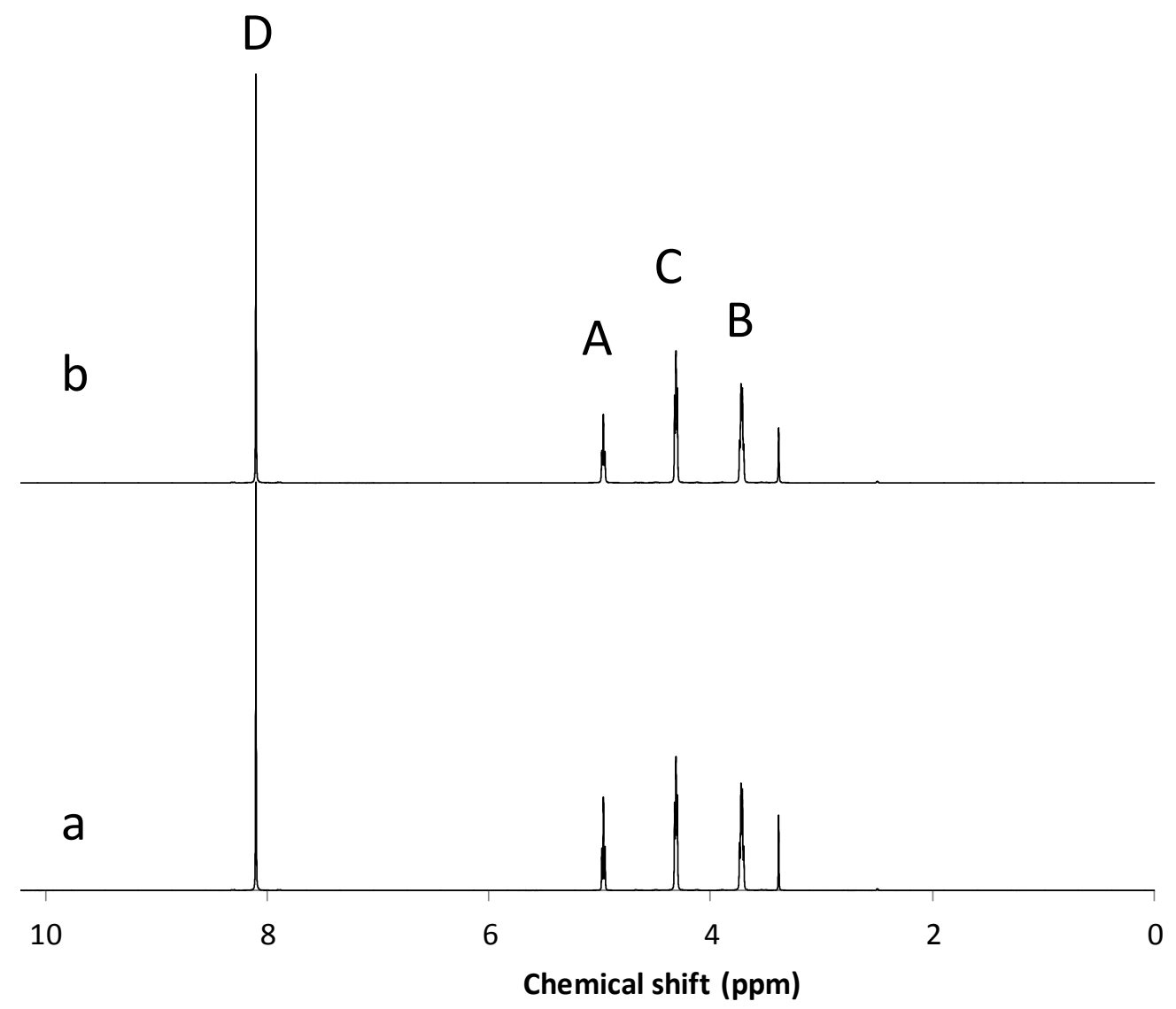

FIGURE 9 NMR spectra of commercial BHET (a) and BHET obtained from glycolysis process with a predegradation step (b). 
TABLE 1: Number average molecular weight $(\mathrm{Mn})$ and weight average molecular weight (Mw) of PETs. The Pre-degraded PET sample was made from compounding PET with Perkalite nanocatalyst and $2 \mathrm{ml}$ EG.

\begin{tabular}{lccc}
\hline Sample Name & $\mathbf{M}_{\mathrm{n}}$ & $\mathbf{M}_{\mathrm{w}}$ & PDI \\
\hline Virgin PET & 4670 & 18620 & 3.98 \\
Pre-degraded PET & 920 & 3830 & 4.16 \\
\hline
\end{tabular}

